\title{
Teaching ESP online during the COVID-19 pandemic: An account of Argentinian students on this teaching modality
}

\author{
Liliana Waicekawsky, ${ }^{1 *}$ Laura Laurenti $^{1}$, and Florencia Yuvero ${ }^{1}$ \\ ${ }^{1}$ National University of San Luis, Ejército de los Andes 950, San Luis, Argentina
}

\begin{abstract}
In today's world, the language par excellence in written and oral communication in all the spheres of commerce, business, education and science is English (Swales, 1990). Undeniably, this is the most widely chosen language for communication in academic settings among nonnative speakers who share neither a common first language nor a common culture to carry out scientific interactions such as delivering a conference or university lecture, submitting a grant proposal, or writing a paper or dissertation, presenting a conference poster, to name just a few (Seildhofer, 2006). In many countries in which English is not the native language, most universities and institutions of higher learning adopt an approach called ESP (English for Specific Purposes) for English language teaching. ESP consists of tailoring language instruction to meet the needs of learners who belong to particular disciplines or professions and studies the language appropriate to such activities. Most universities have included ESP programs as part of their syllabuses because they acknowledge the importance of helping non-native speakers of English "master the functions and linguistic conventions of texts that they need to read and write in their disciplines and professions" (Hyon, 1996, p. 698). At the National University of San Luis, since the first years of any course of study, students are exposed to different genres which are published in English. This means that their exposure to the language starts at an early stage of instruction. In general, classes are face-to-face and in a classroom that is fit to that aim. Unfortunately, due to an unexpected pandemic, instruction was forced to adopt a different course. In this paper, we attempt to give an account of how the teaching of ESP is taking place under the circumstances we are facing due to COVID-19. We have changed our methodology so as to meet the demands of the students and the university alike. We administered a questionnaire to know the perceptions and opinions of our students as regards the material, teacher performance, testing, and online modality adopted. Results suggest that the opinions are divided as regards non-face to face or face-to-face teaching modality. Although many students prefer virtuality because of the time flexibility it provides, others assert that nothing is more valuable than teacher and classmates interaction.
\end{abstract}

\footnotetext{
*Corresponding author: lailawal2018@gmail.com
} 


\section{Introduction}

In today's world, the language par excellence in written and oral communication in all the spheres of commerce, business, education and science is English (Swales, 1990). Undeniably, this is the most widely chosen language for communication in academic settings among non-native speakers who share neither a common first language nor a common culture to carry out scientific interactions such as delivering a conference or university lecture, submitting a grant proposal, or writing a paper or dissertation, presenting a conference poster, to name just a few (Seildhofer, 2006). In fact, in international events, advances in science are generally communicated in English, which reinforces arguments in favor of mastering this language in areas of scientific publication and academic exchange (Ferguson et al., 2011).

In many countries in which English is not the native language, most universities and institutions of higher learning adopt an approach called ESP (English for Specific Purposes) for English language teaching. ESP consists of tailoring language instruction to meet the needs of learners who belong to particular disciplines or professions and studies the language appropriate to such activities. Most universities have included ESP programs as part of their syllabuses because they acknowledge the importance of helping non-native speakers of English "master the functions and linguistic conventions of texts that they need to read and write in their disciplines and professions" (Hyon, 1996, p. 698). This is the teaching approach that the authors of this study have followed.

At the National University of San Luis (Argentina), since the first years of any course of study, students are exposed to different genres which are published in English. This means that their exposure to the language starts at an early stage of instruction. In general, classes are face-to-face and delivered in a classroom that is fit to that aim. Unfortunately, due to the COVID-19 pandemic, instruction was forced to adopt a different course. It was imperative that as teachers we got involved in the "era of invention" that Mariana Maggio (2018) raises for this postmodern university setting that challenges us to "invent the class" (Maggio, $p$ .28). Undoubtedly, Information and Communication Technologies (ICT) became the perfect allies to promote this change.

In this particular setting, we opted for what Graham Stanley (2019) calls remote language teaching, characterized as "the way of teaching a language interactively through videoconference" ( $p$.8). The author clarifies that it involves online teaching that is developed synchronously with students, as a replacement for face-to-face classes. We needed to acquire the necessary didactic knowledge so that the inclusion of technologies could make learning conducive.

After having read about different teaching models that included technologies we chose the Technological Pedagogical Content Knowledge (TPACK) proposed by Koehler \& Mishra (2006), which emphasizes the necessary knowledge that must be involved in order to carry out teaching proposals making effective use of technologies. It points out that both disciplinary and technological knowledge are not enough if pedagogical knowledge is not considered to be able to fertilize a didactic proposal that enhances what each of these areas contributes to the teaching-learning process.

In this paper, we attempt to give an account of how the teaching of ESP is taking place under the circumstances we are facing due to COVID-19. We have changed our methodology so as to meet the demands of the students and the university alike. We administered a questionnaire to know the perceptions and opinions of our students as regards the material, teacher performance, testing, and online modality adopted. Results suggest that the opinions are divided as regards non-face to face or face-to-face teaching modality. Although many students prefer virtuality because of the time flexibility it 
provides, others assert that nothing is more valuable than teacher and classmates interaction.

\subsection{Context}

The English course at the Faculty of Chemistry, Biochemistry and Pharmacy is offered to 12 courses of study divided into 3 learning groups: Group A:.Biology (3th year), Molecular Biology (2th year), Biotechnology (3th year), Food science and technology. (3th year), Biological sciences (2th year) and Biological laboratories (2th year), Group B: Chemical Analyst (1th year), Chemistry (1th year), Chemistry (4th year), Sterilization (3th year) and Group C: Pharmacy (4th year), Biochemistry (5th year) and Food Engineering (5th year). The subject is annual and is taught in-person with a 2-hour weekly workload.

The discursive genres that are approached from an ESP perspective are: handbooks, dictionaries, encyclopedias and abstracts for Groups A and $\mathrm{B}$, and reviews, abstracts and scientific research articles in Group $\mathrm{C}$. The selection of these genres is based on the empirical foundation of the genres students come across and need to decode in order to advance in their studies and educational courses. In the next section, a detailed description of the methodological approach used for online teaching during pandemic is explained.

\section{Methodology}

This mixed method study used a questionnaire for data collection which consisted of 34 questions divided into 7 Sections. We will report here the answers of only 5 sections that serve to account for the objectives set. The questionnaire was created via Google Forms and made available online in the different Facebook groups that the subject English has for communicative and learning purposes at the end of the first semester.

Students were asked to fill in the questionnaire since the Chair was aimed at assessing key aspects related to the learning experience that started in March so as to know how effective the teaching strategies implemented in pandemic had been, among other aspects. It is important to mention that it was not compulsory for them to complete the survey, but highly appreciated. A total of 117 students answered distributed as follows: 49 students from Group A, 31 students from Group B, and 37 students from Group C. As stated above, and in order to attain the objectives of this study, we will only report on Sections 1 and 6 of the questionnaire which account for the online learning experience, Section 2 which asks about the learning material used, Section 4 that inquires about the teachers' performance, and Section 3 that assesses online testing.

\subsection{Sample description}

For this study, we designed a questionnaire which considered different aspects of the teaching-learning experience in the virtual environment. The questionnaire was a Google form designed in Google drive and embedded in each of our Facebook groups. The questions revolved around the online learning experience, the learning material used, teachers' performance and online testing. We have transcribed the questions we posed to our students:

\section{Online learning experience}

1.Do you consider that the virtual means chosen by the professors were useful to study this subject?

2. If your answer was negative, what means do you consider would have helped you to achieve this? 
3. How would you rate your experience in this subject?

4. What is your opinion about this virtual course?

5. What tools did you find most useful?

6. Would you like to go on studying this way?

\section{Learning material used}

1. What were the Class guides like?

2. Could you work on the Class guides on your own?

3. If your answer was negative, who helped you complete them?

4. What is your opinion as regards the videos included in the post-reading section of the guides?

\section{Teacher's performance}

1. How would you rate teachers' performance in the virtual classes?

2. Do you think that teachers' communication was effective?

\section{Online testing}

1. How would you rate the level of difficulty of the Practical Task (PT)?

2. How would you rate the level of difficulty of the First Midterm Exam (FME)?

3. Did you use online dictionaries to perform the evaluations?

4. Did you use paper-based dictionaries to take the assessment?

5. Did you use the Lexico-grammar Guide to carry out the assessment?

All the answers provided by the students were registered in an excel sheet.

\subsection{Online Teaching and learning methodology}

\section{Technological tools used}

Classes were taught synchronously respecting the day and class time that they would have had in person in order to allow a better adaptation of the students to the rhythm of the virtual course. The application that was suggested by the UNSL for the teaching of classes was Google Meet, a free application offered by Google that allows the recording of classes and the participation of up to 100 students. This tool worked very well for teaching classes, recording them, and for asynchronous tutoring classes that were weekly offered. Class attendance was suggested but not compulsory as we knew that Internet access would not be efficient for everyone. Therefore, recording the classes allowed us to make the classes available to the students both for those who had not been able to connect and for those who wanted to see them and review the contents covered.

The means of communication between the students and the teachers was a private Facebook group and email for each group. In addition to making announcements about class dynamics, remembering important dates, Facebook officiated as a Virtual Learning Environment (VLE) since the Class Guides, the resolution of the Class Guides and the Lexical-Grammar Guide were shared there. Besides, links to consultation Web pages (dictionaries, grammar pages) and YouTube videos were also shared as reference material.

\section{Teaching staff}

The teaching staff teach English to the students of the School of Chemistry, Biochemistry and Pharmacy at Universidad Nacional de San Luis (Argentina). The staff is made up of three professors who have a strong background in ESP (English for Specific Purposes). We have extensive experience in teaching reading comprehension to university students and have been working in the university for a long time. We have taken and delivered courses 
in reading comprehension as part of our MA programme in Applied Linguistics. Apart from that, we have also specialized in technology and education.

\section{Results}

\section{About the online learning experience}

There were two sections in the questionnaire that addressed this issue: Section 1 (On the modality of the course) and Section 6 (Opinion on the virtual course). Regarding the former, the students were asked if they considered that the virtual media that the chair had used (Facebook, Meet, Classroom) had served them to study the subject. 96.1\% of the respondents gave an affirmative opinion. In turn, they were asked to rate the virtual course, being the options: Excellent, Good, Fair and Bad. A total of $43.76 .4 \%$ rated the course as Excellent, $48.76 \%$ as Good, and only $4.33 \%$ students chose Fair, clarifying that it was due to her poor Internet connection, not regarding the virtual modality of the subject itself and $3.15 \%$. decided not to answer.

Section 6 takes up a question that serves to validate what was already asked in Section 1 as regards students' opinions about the virtual course offered. Answers were very varied, so we will share those comments that best synthesize students' opinions on this issue:

- It was a different way of studying but it was dynamic and efficient.

$>$ It was a very good experience, the predisposition on the part of the teachers was excellent and it should be noted that it was very organized.

- Class dates were respected, and classes were always excellent. Nothing to complain about.

$>$ I consider that it was the only subject that was more attentive and present for us, the students, during this very atypical situation.

$>$ It was better than other subjects because it was more comprehensive.

- I felt at ease while studying this subject under virtual modality.

- It was excellent for me because I could interact with Professors who explained everything in a clear way. I could also see the videos again and that helped me to study the topics in a better way.

- I am very satisfied. In fact for persons who work and are mothers this modality allows us to attend classes and progress in our careers. I took up three subjects and I really liked the organization of this Chair in particular having respect for the schedule, time and publication of class guides and keys.

- It was great for me (I am mother of two kids and also work, so attending virtual classes made my life easier. I am very satisfied and pleased with virtual modality.

It is possible to perceive the high valuation towards the teachers of the chair, highlighting their good disposition and respect for the class schedule. In turn, the accompaniment of teachers with students in an unconventional course is valued.

Although most of the students lived the virtual experience in a positive way, a small percentage commented on evaluations that denote the initial difficulty of adapting to a virtual learning environment.

$>$ At first it was stressful because it was difficult for me to adapt to the new modality, later I adapted and it was easier for me. 
$>$ At first it was a bit complicated for me, but afterwards I got used to it and lived the experience as if I were in college but from home.

- At first it seemed strange and very different from a face-to-face class, but throughout the course I eventually got used to it.

- It was difficult and complicated at first but the teachers helped overcome all the problems.

- It was fine but I prefer face-to-face classes

- I was not used to virtual classes but I had no problems and could understand the topics easily.

- It was very difficult to be in class in most subjects but the teachers deal with it really well and offer a lot of tools to study.

- It was good but I was left with some doubts.

- It was very hard for me. It is not easy to learn when you depend on internet connection. Face to face classes are better.

- It was difficult for me to adapt to it.

\section{About the learning material}

In relation to this section, students were asked about their opinion regarding the class guides. They could choose from the options: Clear, Confusing, Complete, and Incomplete with an open option if they wanted to provide other appreciations. Students could choose more than one option if they wished. A number of $72.98 \%$ rated the guides as Clear, $23.96 \%$ as Complete, $3.06 \%$ students rated them as very didactic, a bit extensive, confusing, and/or incomplete.

Since we were deeply concerned about students' autonomy when completing the guides we asked whether they were able to complete them on their own. Fortunately, a percentage of $78.7 \%$ answered affirmatively which demonstrated that the guides were well designed and promoted self-directed learning.

Other questions on this axis demanded an assessment of the videos shared in class. This question was divided into two items. The first asked about the videos included in the postreading section of the Guides. Students could choose from Interesting, Boring, Unnecessary, Motivating, Helpful, Never Saw It, and an open option. They were allowed to check more than one option. $36.3 \%$ rated the videos as Interesting, $46.5 \%$ as Useful, $5.1 \%$ as Motivating, $4.3 \%$ as Unnecessary and $8.3 \%$ claimed not to have seen them. In the 2 open responses, on the one hand, one student mentioned that "it was good to try to understand the newscast" and, on the other hand, a student highlighted the fact that the video made the Class Guide longer, which eventually made her abandon this section. Regarding the YouTube videos on grammatical topics, the options were Useful, Unnecessary, Clear, Confusing, Never saw them, and an open response. $73.76 \%$ rated them as Useful, $26.24 \%$ as Clear, and within the 3 open options, 2 of them ratified the usefulness of the videos as they helped them better understand the grammatical issues whereas the other open response mentioned the hope that the videos were more complete.

\section{About teachers' performance}

This section of the questionnaire was particularly relevant for us since it was the first that we delivered the lessons completely online. One of the questions asked students to rate the performance of the teachers during the development of the virtual classes, being the options Poor, Good, Very good and Excellent. A total of $50.1 \%$ considered their performance as Excellent, $40.55 \%$ as Very Good, and $9.35 \%$ as Good.

Another question asked whether students considered that the teachers had communicated efficiently along the course, being the options Yes, No, Sometimes, and an 
open choice for them to fill in if they wanted to add another option. All the students answered affirmatively, without adding further options.

\section{About online testing}

The evaluations during the first semester were a Practical Task (PT) and a First Midterm Exam (FME). For the PT, the Google Classroom application was used. As it did not turn out to be a practical tool and we faced some technical problems which were also acknowledged by some students, for the FME we agreed on using Facebook to post the examination file and asked students to send the file resolved to our email accounts for their correction.

Students' opinions of the evaluations were assessed in Section 3 focusing on the following aspects:

I. How would you rate the degree of difficulty of TP 1 ?

II. How would you rate the degree of difficulty of the First Midterm Exam?

III. Did you use online dictionaries to perform the evaluations?

IV. Did you use a paper-based dictionary to take the assessments?

V. Did you use the Lexical-Grammar Guide to carry out the assessments?

For question I and II, the options as regards the degree of the evaluations were: Easy, Difficult and Medium complexity. A number of $29.7 \%$ rated the TP as Easy, $13.5 \%$ as Difficult, and 56.8\% as Medium complexity. For the FME the results showed 10.8 \%, 13.5\% and $75.7 \%$, respectively. As can be seen in both cases, students considered that the evaluations were neither easy nor difficult, signaling a good balance in the activities proposed to assess learning.

Questions III and IV were expecting a yes and no answer. The answers showed that $73.8 \%$ used an online dictionary and that $26.2 \%$ also used a paper-based dictionary. We were really satisfied that students made use of a reference material that was heavily used in class. Finally, $86.5 \%$ of students answered question $\mathrm{V}$ affirmatively, indicating that the Lexico-grammar Guide was really helpful in their accomplishment of the exam tasks.

\section{Conclusion}

From the beginning of 2020, COVID-19 has affected the way education has developed around the world. Technology is now having a central role as classes take place in virtual learning environments. In this paper, we attempted to give an account of how the teaching of ESP is taking place under the circumstances we are facing. We implemented ESP virtual classes to our university students and we delved into their perceptions as regards classes, materials used, assessment and teachers' performance. Students could verbalize through a questionnaire the effectiveness of virtual classes in developing reading comprehension, in spite of the drawbacks they faced due to lack of good connectivity and impossibility of attending face-to face classes. Moreover, other students highlighted the importance and usefulness of virtual modality to advance in their career in a context where they face the challenge of being mothers, working and studying. In this way, they also remarked the efficient organization of the Chair regarding schedules, class materials and appropriate feedback delivered through virtual platforms. They added that in spite of unstable connection and Internet problems the teachers helped to overcome the possible drawbacks offering them a lot of tools to study and adapting themselves to students' new contexts. Although students have positive attitudes towards learning English through virtual classes because of the time flexibility they provide, others acknowledge that face-to face encounters are the most valuable ones to foster teacher and classmates' interaction. 


\section{Acknowledgments}

This paper was financially supported by the Russian Foundation for Basic Research, grant No. 20-012-22046.

\section{References}

1. G. Ferguson, C. Pérez-Llantada, \& R. Plo, English as an international language of scientific publication: A study of attitudes. World Englishes 30 (1), 41-59 (2011). https://doi.org/10.1111/j.1467-971X.2010.01656.X

2. S. Hyon, Genre in three traditions: Implications for ESL, TESOL Quarterly, 30, 693-722 (1996)

3. M. Maggio, Reinventar la clase en la universidad (2018)

4. P. Mishra, \& M.J. Koehler, Technological pedagogical content knowledge: A framework for teacher knowledge. Teachers College Record, 108(6), 1017-1054 (2006)

5. B. Seidlhofer, English as a lingua franca in the expanding circle: What it isn't. In R. Rubdy \& M. Saraceni (Eds.), English in the world: Global rules, global roles, pp. 40-50, London: Continuum (2006)

6. G. Stanley, Innovations in education, Remote teaching (2019)

7. J. Swales, Genre analysis: English in academic and research settings, Cambridge: Cambridge University Press, (1990) 